\title{
On Some Grüss-Type Fractional Inequalities Using Saigo Fractional Integral Operator
}

\author{
Vaijanath L. Chinchane ${ }^{1}$ and Deepak B. Pachpatte ${ }^{2}$ \\ ${ }^{1}$ Department of Mathematics, Deogiri Institute of Engineering and Management Studies, Aurangabad 431005, India \\ ${ }^{2}$ Department of Mathematics, Dr. Babasaheb Ambedkar Marathwada University, Aurangabad 431004, India
}

Correspondence should be addressed to Vaijanath L. Chinchane; chinchane85@gmail.com

Received 4 May 2014; Revised 17 July 2014; Accepted 24 July 2014; Published 24 August 2014

Academic Editor: Nasser Saad

Copyright (c) 2014 V. L. Chinchane and D. B. Pachpatte. This is an open access article distributed under the Creative Commons Attribution License, which permits unrestricted use, distribution, and reproduction in any medium, provided the original work is properly cited.

\begin{abstract}
The main aim of this paper is to establish some new fractional integral inequalities of Grüss-type via the Saigo fractional integral operator.
\end{abstract}

\section{Introduction}

Grüss inequality is an inequality which establishes a connection between the integral of the product of two functions and the product of the integrals of the two functions. In 1935, Grüss proved the following well-known classical integral inequality; see $[1,2]$.

Theorem 1 (see [2]). Let $f, g:[a, b] \rightarrow R$ be two integrable functions such that $\phi \leq f(x) \leq \Phi$ and $\gamma \leq g(x) \leq \Gamma$ for all $x \in[a, b] ; \phi, \Phi, \gamma$, and $\Gamma$ are constant; then

$$
\begin{aligned}
& \mid \frac{1}{b-a} \int_{a}^{b} f(x) g(x) d x \\
& -\frac{1}{b-a} \int_{a}^{b} f(x) d x \cdot \frac{1}{b-a} \int_{a}^{b} g(x) d x \mid \\
& \quad \leq \frac{1}{4}(\Gamma-\gamma)(\Phi-\phi),
\end{aligned}
$$

where the constant $1 / 4$ is sharp.

In the past decade, many researchers had numerous generalizations, variants, and extensions of Grüss inequality done in literature; to mention a few, see [1-12] and the references cited therein.
Recently, many authors have studied the fractional integral inequalities via Caputo, Riemann-Liouville, and $q$ fractional integral; see $[6,13-21]$. Some authors have studied the Saigo fractional integral operator; for example, we refer the reader to [22-29] and references cited therein. In [19], Dahmani et al. gave the following fractional integral inequality using Riemann-Liouville fractional integral.

Theorem 2 (see [19]). Let $f$ and $g$ be two integrable functions on $[0, \infty)$ satisfying the condition

$$
\begin{gathered}
m \leq f(x) \leq M, \\
p \leq g(x) \leq P \\
m, M, p, P \in R,
\end{gathered}
$$

on $[0, \infty)$. Then, for all $t>0$ and $\alpha>0$, one has

$$
\begin{aligned}
& \left|\frac{t^{\alpha}}{\Gamma(\alpha+1)} J^{\alpha} f g(t)-J^{\alpha} f(t) J^{\alpha} g(t)\right| \\
& \quad \leq\left(\frac{t^{\alpha}}{2 \Gamma(\alpha+1)}\right)^{2}(M-m)(P-p) .
\end{aligned}
$$

In literature few results have been obtained on some fractional integral inequalities using Hadamard fractional integral and Saigo fractional integral operator in [25, 26, 3032]. Our purpose in this paper is to establish some new results using Saigo fractional integral. 


\section{Preliminaries}

We give some definitions and properties which will be used later. For more details, see [28, 33].

Definition 3. A real-valued function $f(x)(x>1)$ is said to be in space $C_{\mu}(\mu \in R)$, if there exists a real number $p>\mu$ such that $f(x)=x^{p} \phi(x)$, where $\phi(x) \in C(0, \infty)$.

Definition 4 (see $[27,28]$ ). Let $\alpha>0, \beta, \eta \in R$; then the Saigo fractional integral $I_{0, x}^{\alpha, \beta, \eta}[f(x)]$ of order $\alpha$ for a real-valued continuous function $f(x)$ is defined by

$$
\begin{aligned}
I_{0, x}^{\alpha, \beta, \eta} & {[f(x)] } \\
= & \frac{x^{-\alpha-\beta}}{\Gamma(\alpha)} \\
& \cdot \int_{0}^{x}(x-t)^{\alpha-1}{ }_{2} F_{1}\left(\alpha+\beta,-\eta ; \alpha ; 1-\frac{t}{x}\right) f(t) d t,
\end{aligned}
$$

where the function ${ }_{2} F_{1}(-)$ in the right-hand side of $(4)$ is the Gaussian hypergeometric function defined by

$$
{ }_{2} F_{1}(a, b ; c ; x)=\sum_{n=0}^{\infty} \frac{(a)_{n}(b)_{n}}{(c)_{n}} \frac{x^{n}}{n !} .
$$

And $(a)_{n}$ is the Pochhammer symbol

$$
\begin{gathered}
(a)_{n}=a(a+1) \cdots(a+n-1)=\frac{\Gamma(a+n)}{\Gamma(a)}, \\
(a)_{0}=1 .
\end{gathered}
$$

For $f(x)=x^{\mu}$ in (4), we have the known result [28] as

$$
I_{0, x}^{\alpha, \beta, \eta}\left[x^{\mu}\right]=\frac{\Gamma(\mu+1) \Gamma(\mu+1-\beta+\eta)}{\Gamma(\mu+1-\beta) \Gamma(\mu+1+\alpha+\eta)} x^{\mu-\beta}
$$

$(\alpha>0, \min (\mu, \mu-\beta+\eta)>-1, x>0)$.

\section{Grüss-Type Fractional Integral Inequality}

In this section, we establish Grüss-type fractional integral inequality involving Saigo fractional integer operator (4), for which we assume the following.

$\left(A_{1}\right)$ There exist two integrable functions $\Phi_{1}(x)$ and $\Phi_{2}(x)$ on $[0, \infty[$, such that

$$
\Phi_{1}(x) \leq u(x) \leq \Phi_{2}(x), \quad \forall x \in[0, \infty[.
$$

$\left(A_{2}\right)$ There exist two integrable functions $\Psi_{1}(x)$ and $\Psi_{2}(x)$ on $[0, \infty[$, such that

$$
\Psi_{1}(x) \leq v(x) \leq \Psi_{2}(x), \quad \forall x \in[0, \infty[
$$

Lemma 5. Suppose that $u$ is an integrable function on $[0, \infty)$ and $\Phi_{1}(x)$ and $\Phi_{2}(x)$ are two integrable functions on $[0, \infty)$. Assume that the condition $\left(A_{1}\right)$ holds.
Then, for all $x>0, \alpha>\max \{0,-\beta\}, \beta<1, \beta-1<\eta<0$, one has

$$
\begin{aligned}
& \frac{\Gamma(1-\beta+\eta)}{\Gamma(1-\beta) \Gamma(1+\alpha+\eta) x^{\beta}} I_{0, x}^{\alpha, \beta, \eta}\left[u^{2}(x)\right]-\left(I_{0, x}^{\alpha, \beta, \eta}[u(x)]\right)^{2} \\
& =\left(I_{0, x}^{\alpha, \beta, \eta}\left[\Phi_{2}(x)\right]-I_{0, x}^{\alpha, \beta, \eta}[u(x)]\right) \\
& \cdot\left(I_{0, x}^{\alpha, \beta, \eta}[u(x)]-I_{0, x}^{\alpha, \beta, \eta}\left[\Phi_{1}(x)\right]\right) \\
& -\frac{\Gamma(1-\beta+\eta)}{\Gamma(1-\beta) \Gamma(1+\alpha+\eta) x^{\beta}} I_{0, x}^{\alpha, \beta, \eta} \\
& \text { - }\left[\left(\Phi_{2}(x)-u(x)\right)\left(u(x)-\Phi_{1}(x)\right)\right] \\
& +\frac{\Gamma(1-\beta+\eta)}{\Gamma(1-\beta) \Gamma(1+\alpha+\eta) x^{\beta}} I_{0, x}^{\alpha, \beta, \eta}\left[\Phi_{1} u(x)\right] \\
& -I_{0, x}^{\alpha, \beta, \eta}\left[\Phi_{1}(x)\right] I_{0, x}^{\alpha, \beta, \eta}[u(x)] \\
& +\frac{\Gamma(1-\beta+\eta)}{\Gamma(1-\beta) \Gamma(1+\alpha+\eta) x^{\beta}} I_{0, x}^{\alpha, \beta, \eta}\left[\Phi_{2} u(x)\right] \\
& -I_{0, x}^{\alpha, \beta, \eta}\left[\Phi_{2}(x)\right] I_{0, x}^{\alpha, \beta, \eta}[u(x)] \\
& +I_{0, x}^{\alpha, \beta, \eta}\left[\Phi_{1}(x)\right] I_{0, x}^{\alpha, \beta, \eta}\left[\Phi_{2}(x)\right] \\
& -\frac{\Gamma(1-\beta+\eta)}{\Gamma(1-\beta) \Gamma(1+\alpha+\eta) x^{\beta}} I_{0, x}^{\alpha, \beta, \eta}\left[\Phi_{1} \Phi_{2}(x)\right] .
\end{aligned}
$$

Proof. Let $u$ be an integrable function on $[0, \infty)$. For all $\rho, \sigma>$ 0 , we have

$$
\begin{aligned}
\left(\Phi_{2}(\sigma)-u(\sigma)\right)\left(u(\rho)-\Phi_{1}(\rho)\right) \\
+\left(\Phi_{2}(\rho)-u(\rho)\right)\left(u(\sigma)-\Phi_{1}(\sigma)\right) \\
\quad-\left(\Phi_{2}(\rho)-u(\rho)\right)\left(u(\rho)-\Phi_{1}(\rho)\right) \\
\quad-\left(\Phi_{2}(\sigma)-u(\sigma)\right)\left(u(\sigma)-\Phi_{1}(\sigma)\right) \\
=u^{2}(\rho)+u^{2}(\sigma)-2 u(\sigma) u(\rho) \\
\quad+\Phi_{2}(\sigma) u(\rho)+\Phi_{1}(\rho) u(\sigma)-\Phi_{1}(\rho) \Phi_{2}(\sigma) \\
\quad+\Phi_{1}(\rho) u(\sigma)+\Phi_{1}(\sigma) u(\rho)-\Phi_{1}(\sigma) \Phi_{2}(\rho) \\
\quad-\Phi_{2}(\rho) u(\rho)+\Phi_{1}(\rho) \Phi_{2}(\rho)-\Phi_{1}(\rho) u(\rho) \\
\quad-\Phi_{2}(\sigma) u(\sigma)+\Phi_{1}(\sigma) \Phi(\sigma)-\Phi_{1}(\sigma) u(\sigma)
\end{aligned}
$$

Consider

$$
\begin{aligned}
G(x, \rho)= & \frac{x^{-\alpha-\beta}(x-\rho)^{\alpha-1}}{\Gamma(\alpha)} \\
& \cdot{ }_{2} F_{1}\left(\alpha+\beta,-\eta ; \alpha ; 1-\frac{\rho}{x}\right)
\end{aligned}
$$




$$
\begin{aligned}
= & \frac{1}{\Gamma(\alpha)} \frac{(x-\rho)^{\alpha-1}}{x^{\alpha+\beta}}+\frac{(\alpha+\beta)(-\eta)}{\Gamma(\alpha+1)} \frac{(x-\rho)^{\alpha}}{x^{\alpha+\beta+1}} \\
& +\frac{(\alpha+\beta)(\alpha+\beta+1)(-\eta)(-\eta+1)}{\Gamma(\alpha+2)} \frac{(x-\rho)^{\alpha+1}}{x^{\alpha+\beta+2}} \\
& +\cdots, \quad(\rho \in(0, x) ; x>0) .
\end{aligned}
$$

Clearly, function $G(x, \rho)$ positive since for all $\rho \in(0, x)(x>$ $0)$. Multiplying both sides of (11) by $G(x, \rho)$, then integrating resulting identity with respect to $\rho$ from 0 to $x$, we get

$$
\begin{aligned}
& \left(\Phi_{2}(\sigma)-u(\sigma)\right)\left(I_{0, x}^{\alpha, \beta, \eta}[u(x)]-I_{0, x}^{\alpha, \beta, \eta}\left[\Phi_{1}(x)\right]\right) \\
& +\left(I_{0, x}^{\alpha, \beta, \eta}\left[\Phi_{2}(x)\right]-I_{0, x}^{\alpha, \beta, \eta}[u(x)]\right)\left(u(\sigma)-\Phi_{1}(\sigma)\right) \\
& -I_{0, x}^{\alpha, \beta, \eta}\left[\left(\Phi_{2}(x)-u(x)\right)\left(u(x)-\Phi_{1}(x)\right)\right] \\
& -\frac{\Gamma(1-\beta+\eta)}{\Gamma(1-\beta) \Gamma(1+\alpha+\eta) x^{\beta}} \\
& \cdot\left(\Phi_{2}(\sigma)-u(\sigma)\right)\left(u(\sigma)-\Phi_{1}(\sigma)\right) \\
& =I_{0, x}^{\alpha, \beta, \eta}\left[u^{2}(x)\right]+u^{2}(\sigma) \frac{\Gamma(1-\beta+\eta)}{\Gamma(1-\beta) \Gamma(1+\alpha+\eta) x^{\beta}} \\
& -2 u(\sigma) I_{0, x}^{\alpha, \beta, \eta}[u(x)]+\Phi_{2}(\sigma) I_{0, x}^{\alpha, \beta, \eta}[u(x)] \\
& +u(\sigma) I_{0, x}^{\alpha, \beta, \eta}\left[\Phi_{1}(x)\right]-\Phi_{2}(\sigma) I_{0, x}^{\alpha, \beta, \eta}\left[\Phi_{1}(x)\right] \\
& +u(\sigma) I_{0, x}^{\alpha, \beta, \eta}\left[\Phi_{2}(x)\right]+\Phi_{1}(\sigma) I_{0, x}^{\alpha, \beta, \eta}[u(x)] \\
& -\Phi_{1}(\sigma) I_{0, x}^{\alpha, \beta, \eta}\left[\Phi_{2}(x)\right]-I_{0, x}^{\alpha, \beta, \eta}\left[\Phi_{2} u(x)\right] \\
& +I_{0, x}^{\alpha, \beta, \eta}\left[\Phi_{1} \Phi_{2}(x)\right]-I_{0, x}^{\alpha, \beta, \eta}\left[\Phi_{1} u(x)\right] \\
& -\Phi_{2}(\sigma) u(\sigma) \frac{\Gamma(1-\beta+\eta)}{\Gamma(1-\beta) \Gamma(1+\alpha+\eta) x^{\beta}} \\
& +\Phi_{1}(\sigma) \Phi_{2}(\sigma) \frac{\Gamma(1-\beta+\eta)}{\Gamma(1-\beta) \Gamma(1+\alpha+\eta) x^{\beta}} \\
& -\Phi_{1}(\sigma) u(\sigma) \frac{\Gamma(1-\beta+\eta)}{\Gamma(1-\beta) \Gamma(1+\alpha+\eta) x^{\beta}} .
\end{aligned}
$$

Multiplying both sides of (13) by $G(x, \sigma)$ and is positive since for all $\sigma \in(0, x)(x>0)$, and defined as in (12). Then integrating resulting identity with respect to $\sigma$ from 0 to $x$, we have

$$
\begin{gathered}
\left(I_{0, x}^{\alpha, \beta, \eta}\left[\Phi_{2}(x)\right]-I_{0, x}^{\alpha, \beta, \eta}[u(x)]\right) \\
\cdot\left(I_{0, x}^{\alpha, \beta, \eta}[u(x)]-I_{0, x}^{\alpha, \beta, \eta}\left[\Phi_{1}(x)\right]\right) \\
+\left(I_{0, x}^{\alpha, \beta, \eta}\left[\Phi_{2}(x)\right]-I_{0, x}^{\alpha, \beta, \eta}[u(x)]\right) \\
\cdot\left(I_{0, x}^{\alpha, \beta, \eta}[u(x)]-I_{0, x}^{\alpha, \beta, \eta}\left[\Phi_{1}(x)\right]\right)
\end{gathered}
$$

$$
\begin{aligned}
& -\frac{\Gamma(1-\beta+\eta)}{\Gamma(1-\beta) \Gamma(1+\alpha+\eta) x^{\beta}} I_{0, x}^{\alpha, \beta, \eta} \\
& \cdot\left[\left(\Phi_{2}(x)-u(x)\right)\left(u(x)-\Phi_{1}(x)\right)\right] \\
& -\frac{\Gamma(1-\beta+\eta)}{\Gamma(1-\beta) \Gamma(1+\alpha+\eta) x^{\beta}} I_{0, x}^{\alpha, \beta, \eta} \\
& \cdot\left[\left(\Phi_{2}(x)-u(x)\right)\left(u(x)-\Phi_{1}(x)\right)\right] \\
& =I_{0, x}^{\alpha, \beta, \eta}\left[u^{2}(x)\right] \frac{\Gamma(1-\beta+\eta)}{\Gamma(1-\beta) \Gamma(1+\alpha+\eta) x^{\beta}} \\
& +I_{0, x}^{\alpha, \beta, \eta}\left[u^{2}(x)\right] \frac{\Gamma(1-\beta+\eta)}{\Gamma(1-\beta) \Gamma(1+\alpha+\eta) x^{\beta}} \\
& -2 I_{0, x}^{\alpha, \beta, \eta}[u(x)] I_{0, x}^{\alpha, \beta, \eta}[u(x)]+I_{0, x}^{\alpha, \beta, \eta}\left[\Phi_{2}(x)\right] I_{0, x}^{\alpha, \beta, \eta}[u(x)] \\
& +I_{0, x}^{\alpha, \beta, \eta}[u(x)] I_{0, x}^{\alpha, \beta, \eta}\left[\Phi_{1}(x)\right] \\
& -I_{0, x}^{\alpha, \beta, \eta}\left[\Phi_{2}(x)\right] I_{0, x}^{\alpha, \beta, \eta}\left[\Phi_{1}(x)\right] \\
& +I_{0, x}^{\alpha, \beta, \eta}[u(x)] I_{0, x}^{\alpha, \beta, \eta}\left[\Phi_{2}(x)\right]+I_{0, x}^{\alpha, \beta, \eta}\left[\Phi_{1}(x)\right] I_{0, x}^{\alpha, \beta, \eta}[u(x)] \\
& -I_{0, x}^{\alpha, \beta, \eta}\left[\Phi_{1}(x)\right] I_{0, x}^{\alpha, \beta, \eta}\left[\Phi_{2}(x)\right] \\
& -I_{0, x}^{\alpha, \beta, \eta}\left[\Phi_{2} u(x)\right] \frac{\Gamma(1-\beta+\eta)}{\Gamma(1-\beta) \Gamma(1+\alpha+\eta) x^{\beta}} \\
& +I_{0, x}^{\alpha, \beta, \eta}\left[\Phi_{1} \Phi_{2}(x)\right] \frac{\Gamma(1-\beta+\eta)}{\Gamma(1-\beta) \Gamma(1+\alpha+\eta) x^{\beta}} \\
& -I_{0, x}^{\alpha, \beta, \eta}\left[\Phi_{1} u(x)\right] \frac{\Gamma(1-\beta+\eta)}{\Gamma(1-\beta) \Gamma(1+\alpha+\eta) x^{\beta}} \\
& -I_{0, x}^{\alpha, \beta, \eta}\left[\Phi_{2} u(x)\right] \frac{\Gamma(1-\beta+\eta)}{\Gamma(1-\beta) \Gamma(1+\alpha+\eta) x^{\beta}} \\
& +I_{0, x}^{\alpha, \beta, \eta}\left[\Phi_{1} \Phi_{2}(x)\right] \frac{\Gamma(1-\beta+\eta)}{\Gamma(1-\beta) \Gamma(1+\alpha+\eta) x^{\beta}} \\
& -I_{0, x}^{\alpha, \beta, \eta}\left[\Phi_{1} u(x)\right] \frac{\Gamma(1-\beta+\eta)}{\Gamma(1-\beta) \Gamma(1+\alpha+\eta) x^{\beta}} .
\end{aligned}
$$

It follows that

$$
\begin{aligned}
2\left(I_{0, x}^{\alpha, \beta, \eta}\left[\Phi_{2}(x)\right]-I_{0, x}^{\alpha, \beta, \eta} u(x)\right) & \cdot\left(I_{0, x}^{\alpha, \beta, \eta} u(x)-I_{0, x}^{\alpha, \beta, \eta}\left[\Phi_{1}(x)\right]\right) \\
& -2 \frac{\Gamma(1-\beta+\eta)}{\Gamma(1-\beta) \Gamma(1+\alpha+\eta) x^{\beta}} I_{0, x}^{\alpha, \beta, \eta} \\
\cdot & {\left[\left(\Phi_{2}(x)-u(x)\right)\left(u(x)-\Phi_{1}(x)\right)\right] }
\end{aligned}
$$




$$
\begin{aligned}
= & 2 I_{0, x}^{\alpha, \beta, \eta}\left[u^{2}(x)\right] \frac{\Gamma(1-\beta+\eta)}{\Gamma(1-\beta) \Gamma(1+\alpha+\eta) x^{\beta}} \\
& -2\left(I_{0, x}^{\alpha, \beta, \eta}[u(x)]\right)^{2}+2 I_{0, x}^{\alpha, \beta, \eta}\left[\Phi_{2}(x)\right] I_{0, x}^{\alpha, \beta, \eta}[u(x)] \\
& +2 I_{0, x}^{\alpha, \beta, \eta}[u(x)] I_{0, x}^{\alpha, \beta, \eta}\left[\Phi_{1}(x)\right] \\
& -2 I_{0, x}^{\alpha, \beta, \eta}\left[\Phi_{2}(x)\right] I_{0, x}^{\alpha, \beta, \eta}\left[\Phi_{1}(x)\right] \\
& -2 I_{0, x}^{\alpha, \beta, \eta}\left[\Phi_{2} u(x)\right] \frac{\Gamma(1-\beta+\eta)}{\Gamma(1-\beta) \Gamma(1+\alpha+\eta) x^{\beta}} \\
& +2 I_{0, x}^{\alpha, \beta, \eta}\left[\Phi_{1} \Phi_{2}(x)\right] \frac{\Gamma(1-\beta+\eta)}{\Gamma(1-\beta) \Gamma(1+\alpha+\eta) x^{\beta}} \\
& -2 I_{0, x}^{\alpha, \beta, \eta}\left[\Phi_{1} u(x)\right] \frac{\Gamma(1-\beta+\eta)}{\Gamma(1-\beta) \Gamma(1+\alpha+\eta) x^{\beta}},
\end{aligned}
$$

which gives (10).

If $\Phi_{1}(x)=p$ and $\Phi_{2}(x)=P, p, P \in R$ for all $x \in[0, \infty[$, then inequality (10) reduces to following lemma.

Lemma 6. Let $p, P \in R$, and $u(x)$ be an integrable function on $[0, \infty)$ satisfying the condition $p \leq u(x) \leq P$. Then for all $x>0, \alpha>\max \{0,-\beta\}, \beta<1, \beta-1<\eta<0$, one has

$$
\begin{aligned}
& \frac{\Gamma(1-\beta+\eta)}{\Gamma(1-\beta) \Gamma(1+\alpha+\eta) x^{\beta}} I_{0, x}^{\alpha, \beta, \eta}\left[u^{2}(x)\right]-\left(I_{0, x}^{\alpha, \beta, \eta}[u(x)]\right)^{2} \\
& =\left(P \frac{\Gamma(1-\beta+\eta)}{\Gamma(1-\beta) \Gamma(1+\alpha+\eta) x^{\beta}}-I_{0, x}^{\alpha, \beta, \eta}[u(x)]\right) \\
& \quad \cdot\left(I_{0, x}^{\alpha, \beta, \eta}[u(x)-p] \frac{\Gamma(1-\beta+\eta)}{\Gamma(1-\beta) \Gamma(1+\alpha+\eta) x^{\beta}}\right) \\
& \quad-\frac{\Gamma(1-\beta+\eta)}{\Gamma(1-\beta) \Gamma(1+\alpha+\eta) x^{\beta}} \\
& \quad \times I_{0, x}^{\alpha, \beta, \eta}[(P-u(x))(u(x)-p)] .
\end{aligned}
$$

Theorem 7. Let $u$ and $v$ be two integrable functions on $[0, \infty)$, and $\Phi_{1}(x), \Phi_{2}(x), \Psi_{1}(x)$, and $\Psi_{2}(x)$ are four integrable functions on $[0, \infty)$ satisfying the conditions $A_{1}$ and $A_{2}$ on $[0, \infty)$. Then for all $x>0, \alpha>\max \{0,-\beta\}, \beta<1, \beta-1<\eta<$ 0 , one has

$$
\begin{aligned}
& \mid \frac{\Gamma(1-\beta+\eta)}{\Gamma(1-\beta) \Gamma(1+\alpha+\eta) x^{\beta}} I_{0, x}^{\alpha, \beta, \eta}[u v(x)] \\
& \quad-I_{0, x}^{\alpha, \beta, \eta}[u(x)] I_{0, x}^{\alpha, \beta, \eta}[v(x)] \mid \\
& \leq \sqrt{T\left(u, \Phi_{1}(x), \Phi_{2}(x)\right) T\left(v, \Psi_{1}(x), \Psi_{2}(x)\right)},
\end{aligned}
$$

where $T(a, b, c)$ is defined by

$T(a, b, c)$

$$
\begin{aligned}
= & \left(I_{0, x}^{\alpha, \beta, \eta}[c(x)]-I_{0, x}^{\alpha, \beta, \eta}[a(x)]\right)\left(I_{0, x}^{\alpha, \beta, \eta}[a(x)]-I_{0, x}^{\alpha, \beta, \eta}[b(x)]\right) \\
& +\frac{\Gamma(1-\beta+\eta)}{\Gamma(1-\beta) \Gamma(1+\alpha+\eta) x^{\beta}} I_{0, x}^{\alpha, \beta, \eta}[b a(x)] \\
& -I_{0, x}^{\alpha, \beta, \eta}[b(x)] I_{0, x}^{\alpha, \beta, \eta}[a(x)] \\
& +\frac{\Gamma(1-\beta+\eta)}{\Gamma(1-\beta) \Gamma(1+\alpha+\eta) x^{\beta}} I_{0, x}^{\alpha, \beta, \eta}[c a(x)] \\
& -I_{0, x}^{\alpha, \beta, \eta}[c(x)] I_{0, x}^{\alpha, \beta, \eta}[a(x)]+I_{0, x}^{\alpha, \beta, \eta}[b(x)] I_{0, x}^{\alpha, \beta, \eta}[c(x)] \\
& +\frac{\Gamma(1-\beta+\eta)}{\Gamma(1-\beta) \Gamma(1+\alpha+\eta) x^{\beta}} I_{0, x}^{\alpha, \beta, \eta}[b c(x)] .
\end{aligned}
$$

Proof. Let $u$ and $v$ be two functions defined on $[0, \infty[$ satisfying the conditions $A_{1}$ and $A_{2}$. Define

$$
\begin{array}{r}
H(\rho, \sigma):=(u(\rho)-u(\sigma))(v(\rho)-v(\sigma)) ; \\
\rho, \sigma \in(0, x), \quad x>0 .
\end{array}
$$

It follows that

$$
\begin{aligned}
H(\rho, \sigma):= & u(\rho) v(\rho)-u(\rho) v(\sigma)-u(\sigma) v(\rho) \\
& +u(\sigma) v(\sigma) .
\end{aligned}
$$

Then, multiplying both sides of $(20)$ by $G(x, \rho)$ which is defined by (12) and is positive because for all $\rho \in(0, x)(x>$ $0)$. Then integrating resulting identity with respect to $\rho$ from 0 to $x$, we have

$$
\begin{gathered}
\frac{x^{-\alpha-\beta}}{\Gamma(\alpha)} \int_{0}^{x}(x-\rho)^{\alpha-1}{ }_{2} F_{1}\left(\alpha+\beta,-\eta ; \alpha ; 1-\frac{\rho}{x}\right) H(\rho, \sigma) d \rho \\
=I_{0, x}^{\alpha, \beta, \eta}[u v(x)]-u(\sigma) I_{0, x}^{\alpha, \beta, \eta}[u(x)] \\
\quad-u(\sigma) I_{0, x}^{\alpha, \beta, \eta}[v(x)]+u(\sigma) v(\sigma) I_{0, x}^{\alpha, \beta, \eta} .
\end{gathered}
$$

Again, multiplying both sides of $(21)$ by $G(x, \sigma)$ which is defined by (12) and is positive because for all $\sigma \in(0, x)(x>0)$ since each term of (12) is positive. Then integrating resulting identity with respect to $\sigma$ from 0 to $x$, we have

$$
\begin{aligned}
& \frac{x^{-\alpha-\beta} x^{-\alpha-\beta}}{\Gamma^{2}(\alpha)} \\
& \quad \iiint_{0}^{x}(x-\rho)^{\alpha-1}(x-\sigma)^{\alpha-1}{ }_{2} F_{1}\left(\alpha+\beta,-\eta ; \alpha ; 1-\frac{\rho}{x}\right) \\
& \quad \cdot{ }_{2} F_{1}\left(\alpha+\beta,-\eta ; \alpha ; 1-\frac{\sigma}{x}\right) H(\rho, \sigma) d \rho d \sigma
\end{aligned}
$$




$$
\begin{gathered}
=2\left(\frac{\Gamma(1-\beta+\eta)}{\Gamma(1-\beta) \Gamma(1+\alpha+\eta) x^{\beta}} I_{0, x}^{\alpha, \beta, \eta}[u v(x)]\right. \\
\left.-I_{0, x}^{\alpha, \beta, \eta}[u(x)] I_{0, x}^{\alpha, \beta, \eta}[v(x)]\right) .
\end{gathered}
$$

Applying the Cauchy-Schwarz inequality to (22), we have

$$
\begin{aligned}
& \left(\frac{\Gamma(1-\beta+\eta)}{\Gamma(1-\beta) \Gamma(1+\alpha+\eta) x^{\beta}} I_{0, x}^{\alpha, \beta, \eta} I_{0, x}^{\alpha, \beta, \eta}[u v(x)]\right. \\
& \left.-I_{0, x}^{\alpha, \beta, \eta}[u(x)] I_{0, x}^{\alpha, \beta, \eta}[v(x)]\right)^{2} \\
& \leq\left(\frac{\Gamma(1-\beta+\eta)}{\Gamma(1-\beta) \Gamma(1+\alpha+\eta) x^{\beta}} I_{0, x}^{\alpha, \beta, \eta} I_{0, x}^{\alpha, \beta, \eta}\left[u^{2}(x)\right]\right. \\
& \left.\quad-\left(\frac{\Gamma(1-\beta+\eta)}{\Gamma(1-\beta) \Gamma(1+\alpha+\eta) x^{\beta}} I_{0, x}^{\alpha, \beta, \beta}[u(x)]\right)^{2}\right) \\
& \quad\left(\begin{array}{l}
\left.-\left(I_{0, x}^{\alpha, \beta, \eta}[v(x)]\right)^{2}\right) . \\
\quad
\end{array}\right.
\end{aligned}
$$

Since $\left(\Phi_{2}(x)-u(t)\right)\left(u(t)-\Phi_{1}(x)\right) \geq 0$ and $\left(\Psi_{2}(x)-v(t)\right)(v(t)-$ $\left.\Psi_{1}(x)\right) \geq 0$, we have

$$
\begin{aligned}
\frac{\Gamma(1-\beta+\eta)}{\Gamma(1-\beta) \Gamma(1+\alpha+\eta) x^{\beta}} I_{0, x}^{\alpha, \beta, \eta} \\
\cdot\left[\left(\Phi_{2}(x)-u(t)\right)\left(u(t)-\Phi_{1}(x)\right)\right] \geq 0, \\
\frac{\Gamma(1-\beta+\eta)}{\Gamma(1-\beta) \Gamma(1+\alpha+\eta) x^{\beta}} I_{0, x}^{\alpha, \beta, \eta} \\
\cdot\left[\left(\Psi_{2}(x)-v(t)\right)\left(v(t)-\Psi_{1}(x)\right)\right] \geq 0 .
\end{aligned}
$$

Thus we have

$$
\begin{aligned}
& \frac{\Gamma(1-\beta+\eta)}{\Gamma(1-\beta) \Gamma(1+\alpha+\eta) x^{\beta}} I_{0, x}^{\alpha, \beta, \eta}\left[u^{2}(x)\right]-\left(I_{0, x}^{\alpha, \beta, \eta}[u(x)]\right)^{2} \\
& \leq\left(I_{0, x}^{\alpha, \beta, \eta}\left[\Phi_{2}(x)\right]-I_{0, x}^{\alpha, \beta, \eta}[u(x)]\right) \\
& \quad \cdot\left(I_{0, x}^{\alpha, \beta, \eta}[u(x)]-I_{0, x}^{\alpha, \beta, \eta}\left[\Phi_{1}(x)\right]\right) \\
& +\frac{\Gamma(1-\beta+\eta)}{\Gamma(1-\beta) \Gamma(1+\alpha+\eta) x^{\beta}} I_{0, x}^{\alpha, \beta, \eta}\left[\Phi_{1} u(x)\right] \\
& -I_{0, x}^{\alpha, \beta, \eta}\left[\Phi_{1}(x)\right] I_{0, x}^{\alpha, \beta, \eta}[u(x)]
\end{aligned}
$$

$$
\begin{aligned}
& +\frac{\Gamma(1-\beta+\eta)}{\Gamma(1-\beta) \Gamma(1+\alpha+\eta) x^{\beta}} I_{0, x}^{\alpha, \beta, \eta}\left[\Phi_{2} u(x)\right] \\
& -I_{0, x}^{\alpha, \beta, \eta}\left[\Phi_{2}(x)\right] I_{0, x}^{\alpha, \beta, \eta}[u(x)] \\
& +I_{0, x}^{\alpha, \beta, \eta}\left[\Phi_{1}(x)\right] I_{0, x}^{\alpha, \beta, \eta}\left[\Phi_{2}(x)\right] \\
& -\frac{\Gamma(1-\beta+\eta)}{\Gamma(1-\beta) \Gamma(1+\alpha+\eta) x^{\beta}} I_{0, x}^{\alpha, \beta, \eta}\left[\Phi_{1} \Phi_{2}(x)\right] \\
& =T\left(u, \Phi_{1}, \Phi_{2}\right) \text {, } \\
& \frac{\Gamma(1-\beta+\eta)}{\Gamma(1-\beta) \Gamma(1+\alpha+\eta) x^{\beta}} I_{0, x}^{\alpha, \beta, \eta}\left[u^{2}(x)\right]-\left(I_{0, x}^{\alpha, \beta, \eta}[u(x)]\right)^{2} \\
& \leq\left(I_{0, x}^{\alpha, \beta, \eta}\left[\Psi_{2}(x)\right]-I_{0, x}^{\alpha, \beta, \eta}[u(x)]\right) \\
& \cdot\left(I_{0, x}^{\alpha, \beta, \eta}[u(x)]-I_{0, x}^{\alpha, \beta, \eta}\left[\Psi_{1}(x)\right]\right) \\
& +\frac{\Gamma(1-\beta+\eta)}{\Gamma(1-\beta) \Gamma(1+\alpha+\eta) x^{\beta}} I_{0, x}^{\alpha, \beta, \eta}\left[\Psi_{1} u(x)\right] \\
& -I_{0, x}^{\alpha, \beta, \eta}\left[\Psi_{1}(x)\right] I_{0, x}^{\alpha, \beta, \eta}[u(x)] \\
& +\frac{\Gamma(1-\beta+\eta)}{\Gamma(1-\beta) \Gamma(1+\alpha+\eta) x^{\beta}} I_{0, x}^{\alpha, \beta, \eta}\left[\Psi_{2} u(x)\right] \\
& -I_{0, x}^{\alpha, \beta, \eta}\left[\Psi_{2}(x)\right] I_{0, x}^{\alpha, \beta, \eta}[u(x)] \\
& +I_{0, x}^{\alpha, \beta, \eta}\left[\Psi_{1}(x)\right] I_{0, x}^{\alpha, \beta, \eta}\left[\Psi_{2}(x)\right] \\
& -\frac{\Gamma(1-\beta+\eta)}{\Gamma(1-\beta) \Gamma(1+\alpha+\eta) x^{\beta}} I_{0, x}^{\alpha, \beta, \eta}\left[\Psi_{1} \Psi_{2}(x)\right] \\
& =T\left(u, \Psi_{1}, \Psi_{2}\right) \text {. }
\end{aligned}
$$

Combining (23) and (25) we get the required inequality (17).

Theorem 8. Suppose that $u$ is an integrable function defined on $\left[0, \infty\left[\right.\right.$ and condition $\left(A_{1}\right)$ holds. Then for all $x>0, \alpha>$ $\max \{0,-\beta\}, \psi>\max \{0,-\phi\}, \beta<1, \beta-1<\eta<0, \phi<1$, $\phi-1<\zeta<0$,

$$
\begin{aligned}
I_{0, x}^{\psi, \phi, \zeta} & {\left[\Phi_{1}(x)\right] I_{0, x}^{\alpha, \beta, \eta}[u(x)] } \\
& +I_{0, x}^{\alpha, \beta, \eta}\left[\Phi_{2}(x)\right] I_{0, x}^{\psi, \phi, \zeta}[u(x)] \\
\geq & I_{0, x}^{\alpha, \beta, \eta}\left[\Phi_{2}(x)\right] I_{0, x}^{\psi, \phi, \zeta}\left[\Phi_{1}(x)\right] \\
& +I_{0, x}^{\alpha, \beta, \eta}[u(x)] I_{0, x}^{\psi, \phi, \zeta}[u(x)] .
\end{aligned}
$$

Proof. From the condition $\left(A_{1}\right)$, for all $\rho, \sigma \geq 0$, we have

$$
\begin{gathered}
\left(\Phi_{2}(\rho)-u(\rho)\right)\left(u(\sigma)-\Phi_{1}(\sigma)\right) \geq 0, \\
\Phi_{2}(\rho) u(\sigma)-\Phi_{2}(\rho) \Phi_{1}(\sigma)-u(\rho) u(\sigma)+u(\rho) \Phi_{1}(\sigma) \geq 0,
\end{gathered}
$$


which implies that

$$
\begin{aligned}
& \Phi_{2}(\rho) u(\sigma)+u(\rho) \Phi_{1}(\sigma) \\
& \quad \geq \Phi_{2}(\rho) \Phi_{1}(\sigma)+u(\rho) u(\sigma)
\end{aligned}
$$

Multiplying both sides of $(28)$ by $G(x, \rho)$ which is defined by (12) and remain positive because for all $\rho \in(0, x)(x>0)$ since each term of (12) is positive. Then integrating resulting identity with respect to $\rho$ from 0 to $x$, we get

$$
\begin{aligned}
& u(\sigma) \frac{x^{-\alpha-\beta}}{\Gamma(\alpha)} \\
& \cdot \int_{0}^{x}(x-\rho)^{\alpha-1}{ }_{2} F_{1}\left(\alpha+\beta,-\eta ; \alpha ; 1-\frac{\rho}{x}\right) \Phi_{2}(\rho) d \rho \\
& +\Phi_{1}(\sigma) \frac{x^{-\alpha-\beta}}{\Gamma(\alpha)} \\
& \cdot \int_{0}^{x}(x-\rho)^{\alpha-1}{ }_{2} F_{1}\left(\alpha+\beta,-\eta ; \alpha ; 1-\frac{\rho}{x}\right) u(\rho) d \rho \\
& \geq \Phi_{1}(\sigma) \frac{x^{-\alpha-\beta}}{\Gamma(\alpha)}{ }_{0}^{x}(x-\rho)^{\alpha-1}{ }_{2} F_{1}\left(\alpha+\beta,-\eta ; \alpha ; 1-\frac{\rho}{x}\right) \Phi_{2}(\rho) d \rho \\
& \quad \cdot \int_{2}(\sigma) \frac{x^{-\alpha-\beta}}{\Gamma(\alpha)}{ }_{2} F_{1}\left(\alpha+\beta,-\eta ; \alpha ; 1-\frac{\rho}{x}\right) u(\rho) d \rho . \\
& \quad \int_{0}^{x}(x-\rho)^{\alpha-1}{ }^{x}(2)
\end{aligned}
$$

Therefore,

$$
\begin{aligned}
& u(\sigma) I_{0, x}^{\alpha, \beta, \eta}\left[\Phi_{2}(x)\right]+\Phi_{1}(\sigma) I_{0, x}^{\alpha, \beta, \eta}[u(x)] \\
& \quad \geq \Phi_{1}(\sigma) I_{0, x}^{\alpha, \beta, \eta}\left[\Phi_{2}(x)\right]+u(\sigma) I_{0, x}^{\alpha, \beta, \eta}[u(x)] .
\end{aligned}
$$

Multiplying (30) by $\left(x^{-\psi-\phi} / \Gamma(\psi)\right)(x-\sigma)^{\psi-1}{ }_{2} F_{1}(\psi+$ $\phi,-\zeta ; \psi ; 1-\sigma / x)(\sigma \in(0, x), x>0)$, which remains positive. Then integrating the resulting identity with respect to $\sigma$ from 0 to $x$, we have

$$
\begin{aligned}
I_{0, x}^{\alpha, \beta, \eta}[ & \left.\Phi_{2}(x)\right] \frac{x^{-\psi-\phi}}{\Gamma(\psi)} \\
& \cdot \int_{0}^{x}(x-\sigma)^{\alpha-1}{ }_{2} F_{1}\left(\psi+\phi,-\zeta ; \psi ; 1-\frac{\sigma}{x}\right) u(\sigma) d \sigma \\
& \cdot I_{0, x}^{\alpha, \beta, \eta}[u(x)] \frac{x^{-\psi-\phi}}{\Gamma(\psi)} \\
& \cdot \int_{0}^{x}(x-\sigma)^{\alpha-1}{ }_{2} F_{1}\left(\psi+\phi,-\zeta ; \psi ; 1-\frac{\sigma}{x}\right) \Phi_{1}(\sigma) d \sigma
\end{aligned}
$$

$$
\begin{aligned}
& \geq I_{0, x}^{\alpha, \beta, \eta}\left[\Phi_{2}(x)\right] \frac{x^{-\psi-\phi}}{\Gamma(\psi)} \\
& \quad \cdot \int_{0}^{x}(x-\sigma)^{\alpha-1}{ }_{2} F_{1}\left(\psi+\phi,-\zeta ; \psi ; 1-\frac{\sigma}{x}\right) \Phi_{1}(\sigma) d \sigma \\
& \quad \cdot I_{0, x}^{\alpha, \beta, \eta}[u(x)] \frac{x^{-\psi-\phi}}{\Gamma(\psi)} \\
& \quad \cdot \int_{0}^{x}(x-\sigma)^{\alpha-1}{ }_{2} F_{1}\left(\psi+\phi,-\zeta ; \psi ; 1-\frac{\sigma}{x}\right) u(\sigma) d \sigma,
\end{aligned}
$$

which gives (26) and proves the theorem.

Remark 9. Suppose that $u$ is an integrable function defined on $[0, \infty[$, such that $p \leq u(x) \leq P$, for all $x \in[0, \infty[$ and $p, P \in$ $R$. Then for all $x>0, \alpha>\max \{0,-\beta\}, \psi>\max \{0,-\phi\}, \beta<$ $1, \beta-1<\eta<0, \phi<1, \phi-1<\zeta<0$, we have

$$
\begin{aligned}
& p \frac{\Gamma(1-\phi+\zeta)}{\Gamma(1-\phi) \Gamma(1+\psi+\zeta) x^{\phi}} I_{0, x}^{\alpha, \beta, \eta} u(x) \\
& \quad+P \frac{\Gamma(1-\beta+\eta)}{\Gamma(1-\beta) \Gamma(1+\alpha+\eta) x^{\beta}} I_{0, x}^{\psi, \phi, \zeta}[u(x)] \\
& \geq P p \frac{\Gamma(1-\beta+\eta) \Gamma(1-\phi+\zeta)}{\Gamma(1-\beta) \Gamma(1+\alpha+\eta) \Gamma(1-\phi) \Gamma(1+\psi+\zeta) x^{\beta} x^{\phi}} \\
& \quad+I_{0, x}^{\alpha, \beta, \eta}[u(x)] I_{0, x}^{\psi, \phi, \zeta}[u(x)] .
\end{aligned}
$$

Theorem 10. Suppose that $u$ and $v$ are two integrable functions defined on $\left[0, \infty\left[\right.\right.$ and suppose that $\left(A_{1}\right)$ and $\left(A_{2}\right)$ hold. Then for all $x>0, \alpha>\max \{0,-\beta\}, \psi>\max \{0,-\phi\}, \beta<1, \beta-1<$ $\eta<0, \phi<1, \phi-1<\zeta<0$, the following inequalities are satisfied:

(a) $I_{0, x}^{\psi, \phi, \zeta}\left[\Psi_{1}(x)\right] I_{0, x}^{\alpha, \beta, \eta}[u(x)]+I_{0, x}^{\alpha, \beta, \eta}\left[\Phi_{2}(x)\right] I_{0, x}^{\psi, \phi, \zeta}[v(x)]$

$$
\begin{aligned}
\geq & I_{0, x}^{\psi, \phi, \zeta}\left[\Psi_{1}(x)\right] I_{0, x}^{\alpha, \beta, \eta}\left[\Phi_{2}(x)\right] \\
& +I_{0, x}^{\alpha, \beta, \eta}[u(x)] I_{0, x}^{\psi, \phi, \zeta}[v(x)] .
\end{aligned}
$$

(b) $I_{0, x}^{\psi, \phi, \zeta}\left[\Phi_{1}(x)\right] I_{0, x}^{\alpha, \beta, \eta}[v(x)]+I_{0, x}^{\alpha, \beta, \eta}\left[\Psi_{2}(x)\right] I_{0, x}^{\psi, \phi, \zeta}[u(x)]$

$$
\begin{aligned}
\geq & I_{0, x}^{\psi, \phi, \zeta}\left[\Phi_{1}(x)\right] I_{0, x}^{\alpha, \beta, \eta}\left[\Psi_{2}(x)\right] \\
& +I_{0, x}^{\psi, \phi, \zeta}[u(x)] I_{0, x}^{\alpha, \beta, \eta}[v(x)] .
\end{aligned}
$$

(c) $I_{0, x}^{\alpha, \beta, \eta}\left[\Phi_{2}(x)\right] I_{0, x}^{\psi, \phi, \zeta}\left[\Psi_{2}(x)\right]+I_{0, x}^{\alpha, \beta, \eta}[u(x)] I_{0, x}^{\psi, \phi, \zeta}[v(x)]$

$$
\begin{aligned}
\geq & I_{0, x}^{\alpha, \beta, \eta}\left[\Phi_{2}(x)\right] I_{0, x}^{\psi, \phi, \zeta}[v(x)] \\
& +I_{0, x}^{\psi, \phi, \zeta}\left[\Psi_{2}(x)\right] I_{0, x}^{\alpha, \beta, \eta}[u(x)] .
\end{aligned}
$$


(d) $I_{0, x}^{\alpha, \beta, \eta}\left[\Phi_{1}(x)\right] I_{0, x}^{\psi, \phi, \zeta}\left[\Psi_{1}(x)\right]+I_{0, x}^{\alpha, \beta, \eta} u(x) I_{0, x}^{\psi, \phi, \zeta}[v(x)]$

$$
\begin{aligned}
\geq & I_{0, x}^{\alpha, \beta, \eta}\left[\Phi_{1}(x)\right] I_{0, x}^{\psi, \phi, \zeta}[v(x)] \\
& +I_{0, x}^{\psi, \phi, \zeta}\left[\Psi_{1}(x)\right] I_{0, x}^{\alpha, \beta, \eta}[u(x)] .
\end{aligned}
$$

Proof. To show (a), we use conditions $\left(A_{1}\right)$ and $\left(A_{2}\right)$, for all $x \in[0, \infty[$; we have

$$
\left(\Phi_{2}(\rho)-u(\rho)\right)\left(v(\sigma)-\Psi_{1}(\sigma)\right) \geq 0
$$

which implies that

$$
\begin{aligned}
& \Phi_{2}(\rho) v(\sigma)+u(\rho) \Psi_{1}(\sigma) \\
& \quad \geq \Phi_{2}(\rho) \Psi_{1}(\sigma)+u(\rho) u(\sigma) .
\end{aligned}
$$

Multiplying both sides of (35) by $G(x, \rho)$, which is defined by (12) and remain positive because for all $\rho \in(0, x)(x>$ 0 ), since each term of (12) is positive. Then integrating the resulting identity with respect to $\rho$ from 0 to $x$, we get

$$
\begin{aligned}
v(\sigma) & \frac{x^{-\alpha-\beta}}{\Gamma(\alpha)} \\
& \cdot \int_{0}^{x}(x-\rho)^{\alpha-1}{ }_{2} F_{1}\left(\alpha+\beta,-\eta ; \alpha ; 1-\frac{\rho}{x}\right) \Phi_{2}(\rho) d \rho \\
& +\Psi_{1}(\sigma) \frac{x^{-\alpha-\beta}}{\Gamma(\alpha)} \\
& \cdot \int_{0}^{x}(x-\rho)^{\alpha-1}{ }_{2} F_{1}\left(\alpha+\beta,-\eta ; \alpha ; 1-\frac{\rho}{x}\right) u(\rho) d \rho \\
\geq & \Psi_{1}(\sigma) \frac{x^{-\alpha-\beta}}{\Gamma(\alpha)}{ }_{2}{ }_{2} F_{1}\left(\alpha+\beta,-\eta ; \alpha ; 1-\frac{\rho}{x}\right) \Phi_{2}(\rho) d \rho \\
& \cdot \int_{0}^{x}(x-\rho)^{\alpha-1}{ }_{2} F_{1}\left(\alpha+\beta,-\eta ; \alpha ; 1-\frac{\rho}{x}\right) u(\rho) d \rho . \\
& \cdot v(\sigma) \frac{x^{-\alpha-\beta}}{\Gamma(\alpha)} \\
& \cdot \int_{0}^{x}(x-\rho)^{\alpha-1}{ }^{x}(36)
\end{aligned}
$$

Therefore,

$$
\begin{aligned}
& v(\sigma) I_{0, x}^{\alpha, \beta, \eta} \Phi_{2}(x)+\Psi_{1}(\sigma) I_{0, x}^{\alpha, \beta, \eta} u(x) \\
& \quad \geq \Psi_{1}(\sigma) I_{0, x}^{\alpha, \beta, \eta} \Phi_{2}(x)+v(\sigma) I_{0, x}^{\alpha, \beta, \eta} u(x) .
\end{aligned}
$$

Multiplying (37) by $\left(x^{-\psi-\phi} / \Gamma(\psi)\right)(x-\sigma)^{\psi-1}{ }_{2} F_{1}(\psi+$ $\phi,-\zeta ; \psi ; 1-\sigma / x)(\sigma \in(0, x), x>0)$, which remains positive.
Then by integrating the resulting identity with respect to $\sigma$ from 0 to $x$, we have

$$
\begin{aligned}
I_{0, x}^{\alpha, \beta, \eta}[ & \left.\Phi_{2}(x)\right] \frac{x^{-\psi-\phi}}{\Gamma(\psi)} \\
& \cdot \int_{0}^{x}(x-\sigma)^{\alpha-1}{ }_{2} F_{1}\left(\psi+\phi,-\zeta ; \psi ; 1-\frac{\sigma}{x}\right) v(\sigma) d \sigma \\
& \cdot I_{0, x}^{\alpha, \beta, \eta}[u(x)] \frac{x^{-\psi-\phi}}{\Gamma(\psi)} \\
& \cdot \int_{0}^{x}(x-\sigma)^{\alpha-1}{ }_{2} F_{1}\left(\psi+\phi,-\zeta ; \psi ; 1-\frac{\sigma}{x}\right) \Psi_{1}(\sigma) d \sigma \\
\geq & I_{0, x}^{\alpha, \beta, \eta}\left[\Phi{ }_{2}(x)\right] \frac{x^{-\psi-\phi}}{\Gamma(\psi)} \\
& \cdot \int_{0}^{x}(x-\sigma)^{\alpha-1}{ }_{2} F_{1}\left(\psi+\phi,-\zeta ; \psi ; 1-\frac{\sigma}{x}\right) \Psi_{1}(\sigma) d \sigma \\
& \cdot I_{0, x}^{\alpha, \beta, \eta}[u(x)] \frac{x^{-\psi-\phi}}{\Gamma(\psi)} \\
& \cdot \int_{0}^{x}(x-\sigma)^{\alpha-1}{ }_{2} F_{1}\left(\psi+\phi,-\zeta ; \psi ; 1-\frac{\sigma}{x}\right) v(\sigma) d \sigma .
\end{aligned}
$$

This gives the desired inequality $(a)$. Using the following condition we prove $(b)-(d)$ :

$$
\begin{aligned}
& \left(\Psi_{2}(\rho)-v(\rho)\right)\left(u(\sigma)-\Phi_{1}(\sigma)\right) \geq 0, \\
& \left(\Phi_{2}(\rho)-u(\rho)\right)\left(v(\sigma)-\Psi_{2}(\sigma)\right) \leq 0, \\
& \left(\Phi_{2}(\rho)-u(\rho)\right)\left(v(\sigma)-\Psi_{1}(\sigma)\right) \leq 0 .
\end{aligned}
$$

Remark 11. Suppose that $u$ and $v$ are two integrable functions defined on $[0, \infty[$. Assume that there exist real constants $P, p, Q$, and $q$ such that

$$
\begin{gathered}
p \leq u(x) \leq P, \\
q \leq v(x) \leq Q, \\
\forall x \in[0, \infty[.
\end{gathered}
$$

Then for all $x>0, \alpha>\max \{0,-\beta\}, \psi>\max \{0,-\phi\}, \beta<1$, $\beta-1<\eta<0, \phi<1, \phi-1<\zeta<0$, the following inequalities are satisfied:

$$
\begin{aligned}
\left(a_{0}\right) q & \frac{\Gamma(1-\phi+\zeta)}{\Gamma(1-\phi) \Gamma(1+\psi+\zeta) x^{\phi}} I_{0, x}^{\alpha, \beta, \eta}[u(x)] \\
& +P I_{0, x}^{\alpha, \beta, \eta} I_{0, x}^{\psi, \phi, \zeta}[v(x)] \\
\geq & q P \frac{\Gamma(1-\beta+\eta) \Gamma(1-\phi+\zeta)}{\Gamma(1-\beta) \Gamma(1+\alpha+\eta) \Gamma(1-\phi) \Gamma(1+\psi+\zeta) x^{\beta} x^{\phi}} \\
& +I_{0, x}^{\alpha, \beta, \eta}[u(x)] I_{0, x}^{\psi, \phi, \zeta}[v(x)] .
\end{aligned}
$$




$$
\begin{aligned}
& \left(b_{0}\right) p \frac{\Gamma(1-\beta+\eta)}{\Gamma(1-\beta) \Gamma(1+\alpha+\eta) x^{\beta}} I_{0, x}^{\alpha, \beta, \eta}[v(x)] \\
& +Q \frac{\Gamma(1-\beta+\eta)}{\Gamma(1-\beta) \Gamma(1+\alpha+\eta) x^{\beta}} I_{0, x}^{\psi, \phi, \zeta}[u(x)] \\
& \geq p Q \frac{\Gamma(1-\beta+\eta) \Gamma(1-\phi+\zeta)}{\Gamma(1-\beta) \Gamma(1+\alpha+\eta) \Gamma(1-\phi) \Gamma(1+\psi+\zeta) x^{\beta} x^{\phi}} \\
& +I_{0, x}^{\psi, \phi, \zeta}[u(x)] I_{0, x}^{\alpha, \beta, \eta}[v(x)] . \\
& \left(c_{0}\right) P Q \frac{\Gamma(1-\beta+\eta) \Gamma(1-\phi+\zeta)}{\Gamma(1-\beta) \Gamma(1+\alpha+\eta) \Gamma(1-\phi) \Gamma(1+\psi+\zeta) x^{\beta} x^{\phi}} \\
& +I_{0, x}^{\alpha, \beta, \eta} u(x) I_{0, x}^{\psi, \phi, \zeta}[v(x)] \\
& \geq P \frac{\Gamma(1-\beta+\eta)}{\Gamma(1-\beta) \Gamma(1+\alpha+\eta) x^{\beta}} I_{0, x}^{\psi, \phi, \zeta}[v(x)] \\
& +p \frac{\Gamma(1-\phi+\zeta)}{\Gamma(1-\phi) \Gamma(1+\psi+\zeta) x^{\phi}} I_{0, x}^{\alpha, \beta, \eta}[u(x)] . \\
& \left(d_{0}\right) p q \frac{\Gamma(1-\phi+\zeta)}{\Gamma(1-\phi) \Gamma(1+\psi+\zeta) x^{\phi}} \\
& +I_{0, x}^{\alpha, \beta, \eta}[u(x)] I_{0, x}^{\psi, \phi, \zeta}[v(x)] \\
& \geq p \frac{\Gamma(1-\phi+\zeta)}{\Gamma(1-\phi) \Gamma(1+\psi+\zeta) x^{\phi}} I_{0, x}^{\psi, \phi, \zeta}[v(x)] \\
& +q \frac{\Gamma(1-\phi+\zeta)}{\Gamma(1-\phi) \Gamma(1+\psi+\zeta) x^{\phi}} I_{0, x}^{\alpha, \beta, \eta}[u(x)] \text {. }
\end{aligned}
$$

It is noted that the results give some contributions to the theory of integral inequalities and fractional calculus. Moreover, they are expected to lead to some applications for establishing uniqueness of solutions in fractional differential equations.

\section{Conflict of Interests}

The authors declare that they have no conflict of interests.

\section{Authors' Contribution}

All authors have equal contributions. All authors read and approved the final paper.

\section{References}

[1] G. Grüss, "Uber das Maximum des absoluten Betrages von $(1 /(b-a)) \int_{a}^{b} f(x) g(x) d x-\left(1 /(b-a)^{2}\right) \int_{a}^{b} f(x) d x \int_{a}^{b} g(x) d x$, Mathematische Zeitschrift, vol. 39, no. 1, pp. 215-226, 1935.

[2] Y. Miao, F. Han, and J. Mu, "A new Ostrowski-Grüss type inequality," Kragujevac Journal of Mathematics, vol. 37, no. 2, pp. 307-317, 2013.
[3] S. S. Dragomir, "Some integral inequalities of Grüss type," Indian Journal of Pure and Applied Mathematics, vol. 31, no. 4, pp. 397-415, 2002.

[4] S. S. Dragomir, "A companion of the Grüss inequality and applications," Applied Mathematics Letters, vol. 17, no. 4, pp. 429-435, 2004.

[5] S. S. Dragomir and S. Wang, "An inequality of OstrowskiGrüss'type and its applications to the estimation of error bounds for some special means and for some numerical quadrature rules," Computers and Mathematics with Applications, vol. 33, no. 11, pp. 15-20, 1997.

[6] N. Elezovic, L. Marangunic, and J. Pecaric, "Some improvements of Grüss type inequality," Journal of Mathematical Inequalities, vol. 1, no. 3, pp. 425-436, 2007.

[7] A. M. Mercer and P. R. Mercer, "New proofs of the Grüss inequality," The Australian Journal of Mathematical Analysis and Applications, vol. 1, no. 2, article 12, 2004.

[8] A. M. Mercer, "An improvement of the Gruss inequality," Journal of Inequalities in Pure and Applied Mathematics, vol. 6, no. 4, article 93, 2005.

[9] B. G. Pachpatte, "On Grüss type integral inequalities," Journal of Inequalities in Pure and Applied Mathematics, vol. 3, no. 2, article 27, 2002.

[10] B. G. Pachpatte, "A note on Chebychev-Grüss type inequalities for differentiable functions," Tamsui Oxford Journal of Mathematical Sciences, vol. 22, no. 1, pp. 29-36, 2006.

[11] G. H. Peng and Y. Miao, "A note on Grüss type inequality," Applied Mathematical Sciences, vol. 3, no. 8, pp. 399-402, 2009.

[12] C. Zhu, W. Yang, and Q. Zhao, "Some new fractional $q$ integral Grüss-type inequalities and other inequalities," Journal of Inequalities and Applications, vol. 2012, article 299, 2012.

[13] S. Belarbi and Z. Dahmani, "On some new fractional integral inequalities," Journal of Inequalities in Pure and Applied Mathematics, vol. 10, article 86, no. 3, 5 pages, 2009.

[14] Z. Dahmani, "On Minkowski and Hermite-Hadamard integral inequalities via fractional integration," Annals of Functional Analysis, vol. 1, no. 1, pp. 51-58, 2010.

[15] Z. Dahmani, "New inequalities in fractional integrals," International Journal of Nonlinear Science, vol. 9, no. 4, pp. 493-497, 2010.

[16] Z. Dahmani, “The Riemann-Liouville operator to generate some new inequalities," International Journal of Nonlinear Science, vol. 12, no. 4, pp. 452-455, 2011.

[17] Z. Dahmani, "Some results associated with fractional integrals involving the extended Chebyshev functional," Acta Universitatis Apulensis: Mathematics: Informatics, no. 27, pp. 217-224, 2011.

[18] Z. Dahmani and A. Benzidane, "New inequalities using fractional q-integrals theory," Bulletin of Mathematical Analysis and Applications, vol. 4, no. 1, pp. 190-196, 2012.

[19] Z. Dahmani, L. Tabharit, and S. Taf, "New generalisations of Gruss inequality using Riemann-Liouville fractional integrals," Bulletin of Mathematical Analysis and Applications, vol. 2, no. 3, pp. 92-99, 2010.

[20] Z. Denton and A. S. Vatsala, "Fractional integral inequalities and applications," Computers \& Mathematics with Applications, vol. 59, no. 3, pp. 1087-1094, 2010.

[21] W. Yang, "Some new fractional quantum integral inequalities," Applied Mathematics Letters, vol. 25, no. 6, pp. 963-969, 2012.

[22] V. Kiryakova, "On two Saigo's fractional integral operators in the class of univalent functions," Fractional Calculus \& Applied Analysis, vol. 9, no. 2, pp. 159-176, 2006. 
[23] V. S. Kiryakova, Generalized Fractional Calculus and Applications, vol. 301 of Pitman Research Notes in Mathematics Series, Longman Scientific and Technical, Harlow, UK, 1994.

[24] A. R. Prabhakaran and K. S. Rao, "Saigo operator of fractional integration of hypergeometric functions," International Journal of Pure and Applied Mathematics, vol. 81, no. 5, pp. 755-763, 2012.

[25] S. D. Purohit and R. K. Yadav, "On generalized fractional $q$-integral operators involving the $q$-Gauss hypergeometric function," Bulletin of Mathematical Analysis and Applications, vol. 2, no. 4, pp. 35-44, 2010.

[26] S. D. Purohit and R. K. Raina, "Chebyshev type inequalities for the Saigo fractional integrals and their q-analogues," Journal of Mathematical Inequalities, vol. 7, no. 2, pp. 239-249, 2013.

[27] R. K. Raina, "Solution of Abel-type integral equation involving the APPell hypergeometric function," Integral Transforms and Special Functions, vol. 21, no. 7, pp. 515-522, 2010.

[28] M. Saigo, "A remark on integral operators involving the Gaüss hypergeometric functions," Mathematical Reports of College of General Education: Kyushu University, vol. 11, no. 2, pp. 135-143, 1978.

[29] N. Virchenko, O. Lisetska, and S. L. Kalla, "On some fractional integral operators involving generalized Gauss hypergeometric functions," Applications and Applied Mathematics, vol. 5, no. 10, pp. 1418-1427, 2010.

[30] V. L. Chinchane and D. B. Pachpatte, "A note on some integral inequalities via Hadamard integral," Journal of Fractional Calculus and Applications, vol. 4, no. 11, pp. 1-5, 2013.

[31] V. L. Chinchane and D. B. Pachpatte, "On some integral inequalities using Hadamard fractional integral," Malaya Journal of Matematik, vol. 1, no. 1, pp. 62-66, 2012.

[32] V. L. Chinchane and D. B. Pachpatte, "Some new integral inequalities using Hadamard fractional integral operator," Advances in Inequalities and Applications, vol. 2014, Article ID 12, 2014.

[33] A. A. Kilbas, H. M. Srivastava, and J. J. Trujillo, Theory and Application of Fractional Differential Equations, Elsevier, Amersterdam, The Netherlands, 2006. 


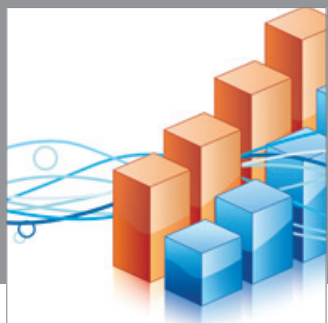

Advances in

Operations Research

mansans

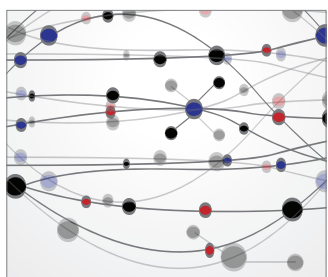

The Scientific World Journal
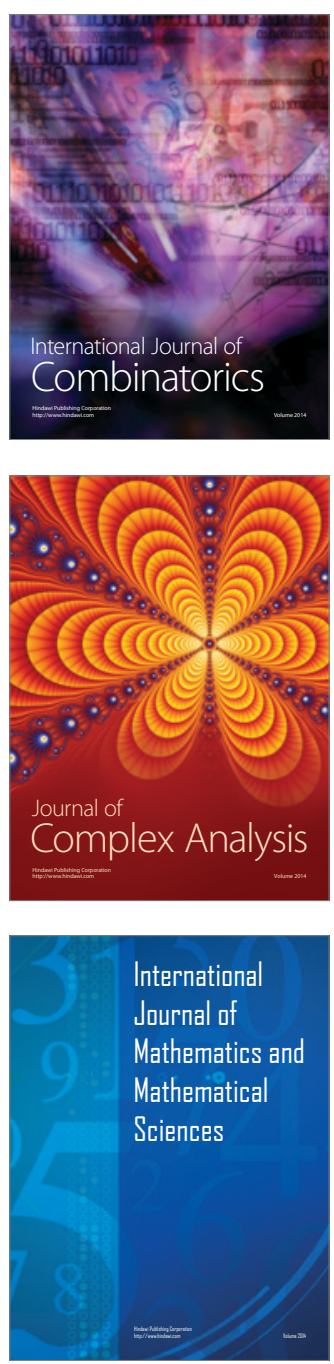
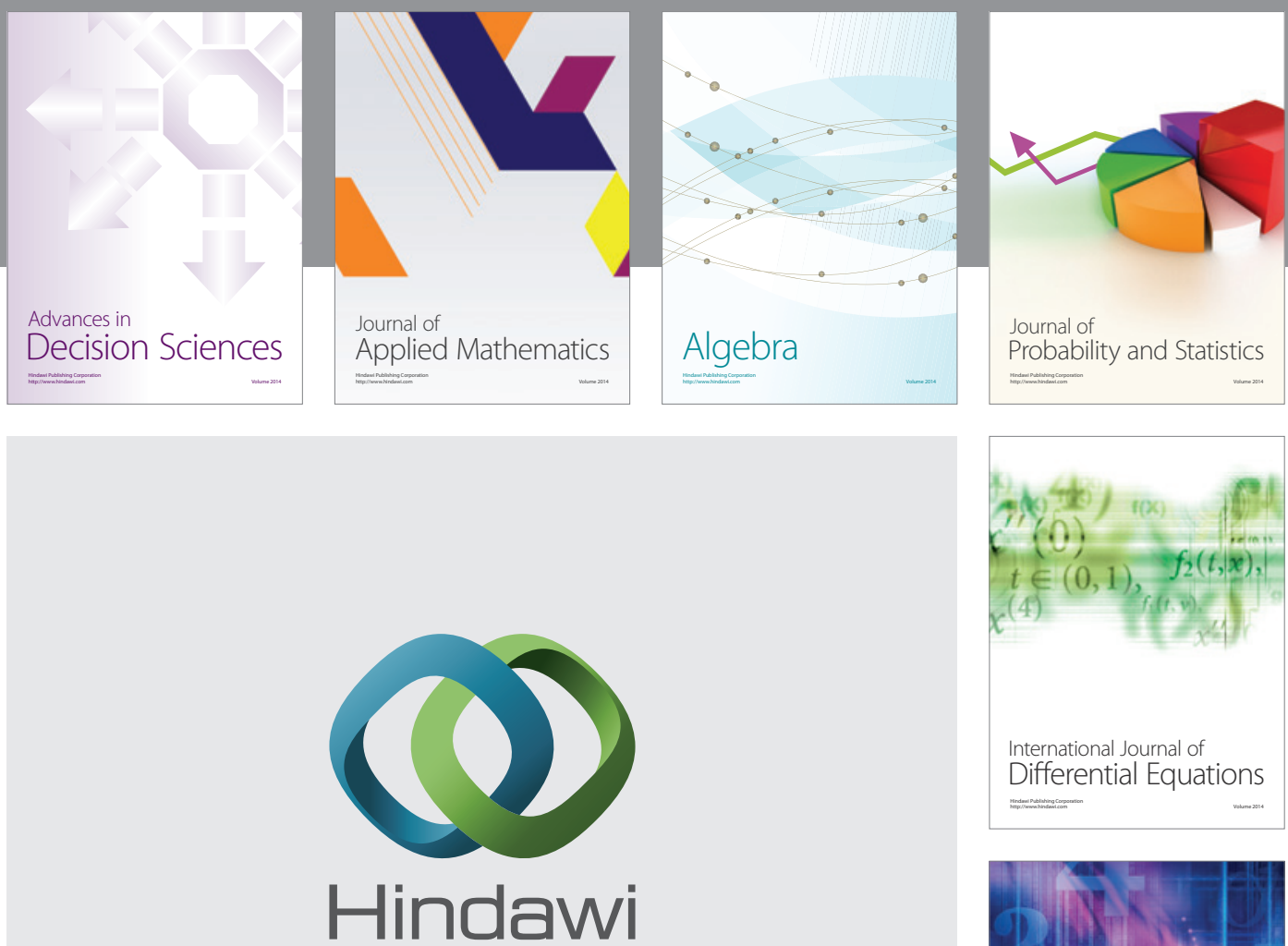

Submit your manuscripts at http://www.hindawi.com
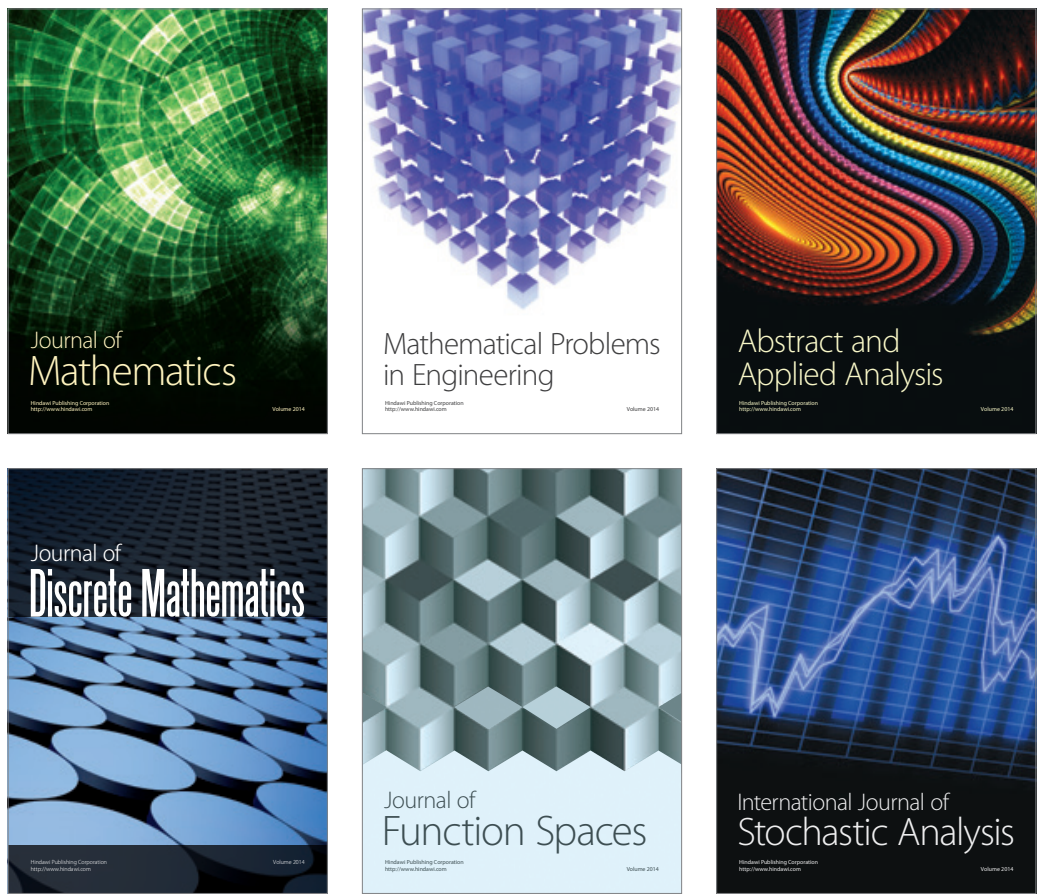

Journal of

Function Spaces

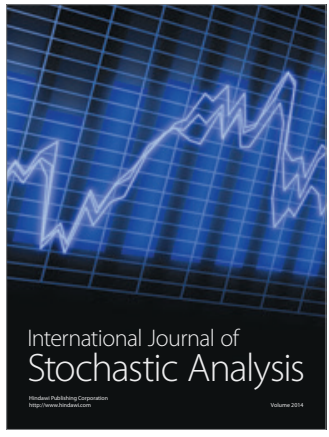

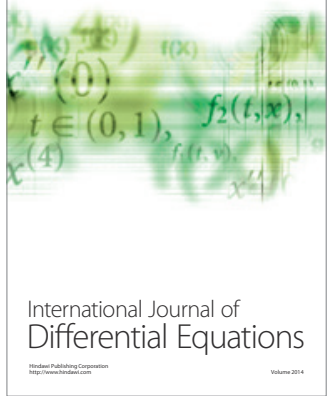
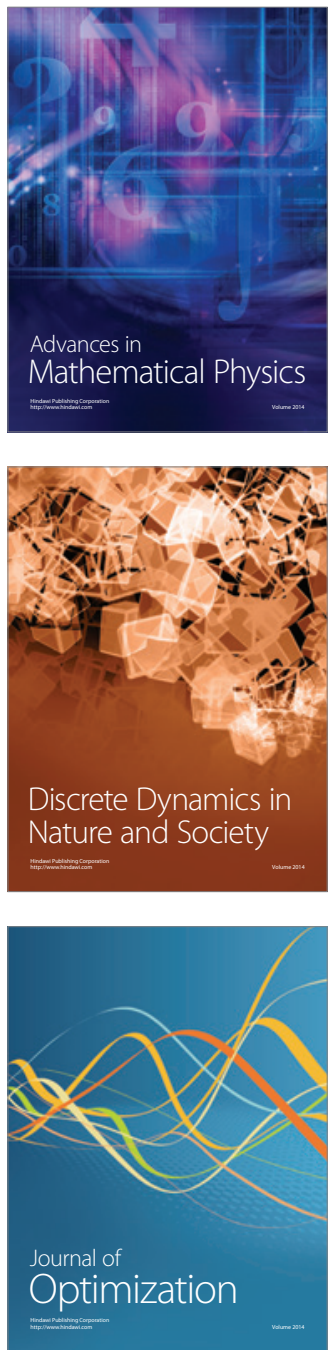